\title{
Solubility of Organics in Triethylene Glycol: Phase Equilibrium Modelling Using the Dortmund and LYNGBY Modified UNIFAC Models
}

\author{
Edison Muzenda, Mohamed Belaid, and Corina Mateescu
}

\begin{abstract}
This work is an attempt to predict the solubility of 60 volatile organic compounds (VOCs) in triethylene glycol aimed at thermodynamically testing the suitability of this solvent as an absorbent for the selected organics. The VOC main groups studied were alkanes, alkenes, alkynes, aldehydes, carboxylic acids and alcohols. The Modified UNIFAC Dortmund and Lyngby models were to study the required phase equilibrium as a function of temperature and composition. Triethylene glycol was found to be suitable for the absorption of low molecular weight aldehydes, alcohols and carboxylic acids. Generally, the infinite dilution activity coefficients computed in this study were low (below 100) indicating that the polymeric solvent studied in this work gave favourable phase equilibrium compared to water, the common industrial solvent. The solubility of VOCs was also found to decrease with increase in solute molecular weight. Compared with literature findings, the Dortmund performed better than the Lyngby procedure. However both models failed to accurately predict phase equilibrium behaviour. The authors therefore agree with literature findings that a specialised group interaction needs to be created for this solvent in the UNIFAC models in order to satisfactorily predict activity coefficients for the studied binary interactions.
\end{abstract}

Index Terms-Absorbent, phase equilibria, solubility, volatile organic compounds.

\section{INTRODUCTION}

VOCs are the common air pollutants emitted mainly by the chemical and petrochemical industries [1]. Emissions of VOCs originate from breathing and loading losses from storage tanks, venting of process vessels, and heat exchange systems [1]. VOCs have adverse effects on humans and the environment. VOCs can affect the health of humans and animals, climate change as well as the growth and decay of plants. Exposure to aromatic hydrocarbons found in soot and tar have been reported to cause cancers [1]. Photochemical smog, which is hazardous to the environment, occurs due to photochemical oxidation involving hydrocarbons and nitrogen oxides in the presence of sunlight. Industries have to

Manuscript received March 12, 2014; revised June 25, 2014. This work was supported by the Universities of Johannesburg and South Africa,

E. Muzenda and M. Belaid are with the Department of Chemical Engineering, Faculty of Engineering and the Built Environment, University of Johannesburg, Doornfontein, Johannesburg 2028, South Africa (tel: 0027-11-5596817; fax:0027-11-5596430; e-mail: emuzenda@uj.ac.za, mbelaid@uj.ac.za).

C. Mateescu is with the Department of Chemical Engineering, College of Science, Engineering and Technology, University of South Africa, Cnr Christian de Wet and Pioneer Avenue, P O Box 392, UNISA 0003, South Africa (e-mail: mateecm@unisa.ac.za). find ways to reduce the emission of volatile organic compounds to meet international regulations. VOCs abatement can be achieved through process and equipment modifications and add-on-control techniques. Add-on control techniques are further classified into destruction and recovery. This study focused on the thermodynamics of absorption, in particular the phase equilibrium or thermodynamic interactions of selected volatile organic compounds and triethylene glycol. Accurate phase equilibrium measurements are quite expensive and tedious. Group contribution methods such as the UNIFAC are useful for preliminary design of separation processes such as absorption and distillation. Ten VOCs in each of the six functional groups, namely alkanes, alkenes, alkynes, aldehydes, alcohols and carboxylic acids were selected for this study. The modified UNIFAC (Dortmund) and (Lyngby) procedures were used to compute the required phase equilibrium. Water the most common industrial solvent has limitations on the absorption of volatile organic compounds as it quickly saturates particularly for high molecular weight organics. For example the infinite dilution activity coefficient of $n$-decane in water is the order of $10^{8}$ [2].

The solvent thermodynamically studied in this work for the abatement of volatile organic compounds through physical absorption triethylene glycol is an alcohol. It is a protic solvent with a hydrogen atom attached to a strongly electronegative atom. Hydrogen bonds are strong dipole dipole attractions between hydrogen atoms that are bonded to highly electronegative atoms. It is a transparent, colourless, low volatility, moderately-viscous, water-soluble liquid. It has no detectable odour under normal conditions with a slightly sweet odour at high vapour concentrations.

The phase equilibrium fundamentals, the modified UNIFAC Dortmund group contribution method, relevant previous studies of interest and computational procedure have been previously discussed [3]-[10]. Recent phase equilibrium measurements using gas - liquid chromatographic techniques for polymeric systems have been discussed [3], [4], [11].

\section{COMPUTATIONAL PRocedurE}

\section{A. Assumptions}

Both the Modified UNIFAC Dortmund and Lyngby are based on assumptions some of which are:

1) The logarithm of the activity coefficient is the sum of the combinatorial part which takes into account the shape and size of the molecule (entropic) plus the residual part accounting for energetic (enthalpic) interactions. 
2) Solutions are composed of functional groups rather than molecules as number of functional or structural groups that make up compounds is much smaller.

3) The residual part resulting from group interactions is assumed to be the difference between the sum of the individual contributions of each solute group in the solution and the sum of the individual contribution in the pure component environment.

4) The individual group contributions in a given environment are a function of group concentrations and temperatures only.

5) The interaction parameters are not symmetrical ( $\Psi n m \neq$ $\Psi m n$ ).

\section{B. Modified UNIFAC Dortmund Model}

Summary of all equations

$$
\begin{gathered}
r_{i}=\sum_{k} v_{k}^{i} R_{k} \\
q_{i}=\sum_{i} v_{i}^{(i)} Q_{k} \\
\phi_{i}^{\prime}=\frac{r_{j}^{3 / 4}}{\sum_{j} x_{j} r_{j}^{3 / 4}} \\
\phi_{i}=\frac{x_{i} r_{i}}{\sum_{i} x_{i} r_{i}} \\
\theta_{i}=\frac{x_{i} q_{i}}{\sum_{i} x_{i} q_{i}}
\end{gathered}
$$

The combinatorial contribution is calculated as in (6)

$$
\begin{gathered}
\ln \gamma_{i}^{C}=1-\phi_{i}^{\prime}+\ln \phi_{i}^{\prime}-5 \times q_{i}\left(\ln \frac{\phi_{i}}{\theta_{i}}+1-\frac{\phi_{i}}{\theta_{i}}\right) \\
\psi_{n m}=\exp \left(-\frac{a_{n m}+b_{n m} T+c_{n m} T^{2}}{T}\right) \\
X_{m}=\frac{\sum_{i} v_{m}^{i} x_{i}}{\sum_{i} v_{n}^{i} x_{i}} \\
\theta_{m}=\frac{X_{m} Q_{m}}{\sum_{m} X_{m} Q_{m}}
\end{gathered}
$$

The residual part is calculated as in (10)

$$
\ln \Gamma_{k}=Q_{k}\left[1-\ln \left(\sum_{m} \theta_{m} \psi_{m k}\right)-\sum_{m} \frac{\theta_{m} \psi_{m k}}{\sum_{n} \theta_{n} \psi_{n m}}\right]
$$

$$
\begin{gathered}
\ln \Gamma_{k}^{(i)}=Q_{k}\left[1-\ln \left(\sum_{m} \theta_{m}^{(i)} \psi_{m k}\right)-\sum_{m} \frac{\theta_{m}^{(i)} \psi_{m k}}{\sum_{n} \theta_{n}^{(i)} \psi_{n m}}\right] \\
\ln \gamma_{1}^{R}=\sum_{k} v_{k}^{(i)}\left[\ln \Gamma_{k}-\ln \Gamma_{k}^{(i)}\right]
\end{gathered}
$$

The activity coefficient is computed using (13)

$$
\ln \gamma_{i}=\ln \gamma_{i}^{C}+\ln \gamma_{i}^{R}
$$

\section{Modified UNIFAC Lyngby Model}

The summary of equations and steps is as follows:

Temperature dependence parameter $\Psi n m$

$\psi_{n m}=\exp \left(-\frac{a_{n m}+b_{n m}\left(T-T_{0}\right)+c_{n m}\left(T \ln \frac{T_{0}}{T}+T-T_{0}\right)}{T}\right)$

The group interaction parameters $a_{n m}, b_{n m}, c_{n m}$ and $a_{m n}, b_{m n}$, $c_{m n}$ are obtained from the Dortmund Data Bank.

$$
X_{m}^{(i)}=\frac{\sum_{j} v_{j}^{m} x_{j}}{\sum_{j} \sum_{n} v_{n . j} x_{j}}
$$

$$
\begin{gathered}
\ln \Gamma_{k}^{(i)}=Q_{k}\left[1-\ln \left(\sum_{m} \theta_{m}^{(i)} \psi_{m k}\right)-\sum_{m} \frac{\theta_{m}^{(i)} \psi_{m k}}{\sum_{n} \theta_{n}^{(i)} \psi_{n m}}\right] \\
X_{m}=\frac{\sum_{j} v_{j}^{m} x_{j}}{\sum_{j} \sum_{n} v_{n . j} x_{j}} \\
\Theta_{m}=\frac{Q_{m} X_{m}}{\sum_{n} Q_{n} X_{n}}
\end{gathered}
$$

The residual activity contribution of group $k$ in the mixture, $\Gamma_{k}$, is calculated using (20).

$$
\ln \Gamma_{k}=Q_{k}\left[1-\ln \left(\sum_{m} \Theta_{m} \Psi_{m k}\right)-\sum_{m} \frac{\Theta_{m} \Psi_{k m}}{\sum_{n} \Theta_{n} \Psi_{n m}}\right]
$$

The residual contribution to the activity coefficient is calculated as: 


$$
\ln \gamma_{i}^{R}=\sum v_{k}^{(i)}\left(\ln \Gamma_{k}-\ln \Gamma_{k}^{(i)}\right)
$$

The van der Waals volume is determined using the group volumes, $R_{k}$ in (22)

$$
r_{i}=\sum_{k} v_{k}^{(i)} R_{k}
$$

The segment fraction $\omega_{i}$ is calculated using (23)

$$
\omega_{i}=\frac{x_{i} r_{i}^{2 / 3}}{\sum_{j} x_{j} r_{j}^{2 / 3}}
$$

The combinatorial contribution to the activity coefficients is computed using (24)

$$
\ln \gamma_{i}^{C}=\ln \frac{\omega_{i}}{x_{i}}+1-\frac{\omega_{i}}{x_{i}}
$$

\section{RESULTS AND DISCUSSION}

A. Alkanes

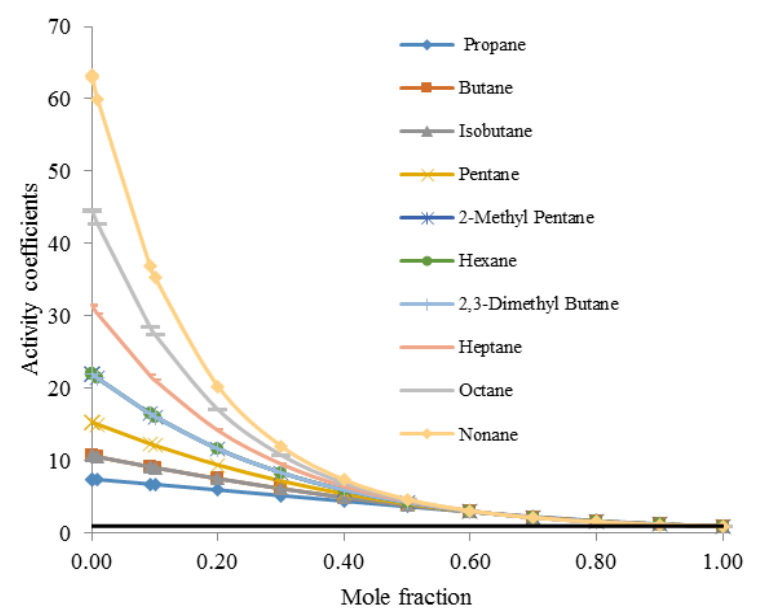

Fig. 1. Variation of Infinite dilution activity coefficients with mole fraction (Modified UNIFAC Dortmund).

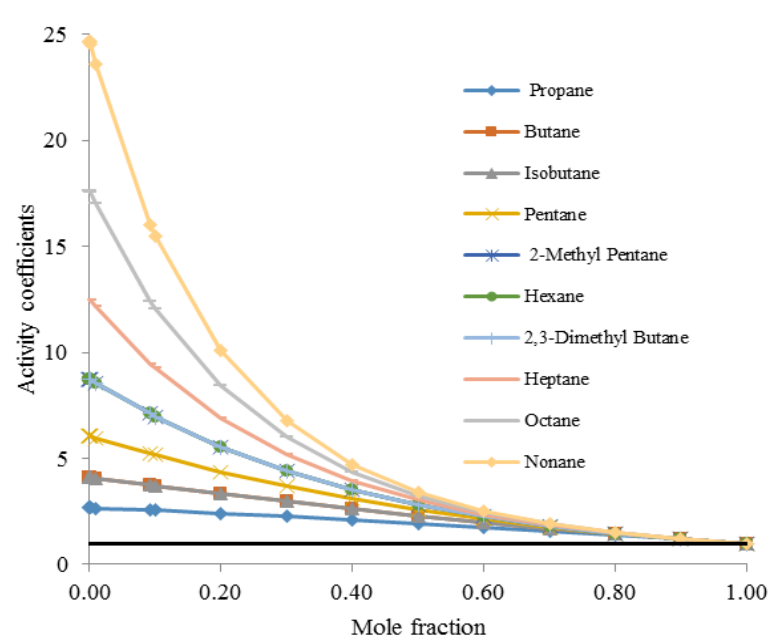

Fig. 2. Variation of Infinite dilution activity coefficients with mole fraction (Modified UNIFAC Lyngby).
For alkanes, Fig. 1 and Fig. 2, the solubility decreases with increasing VOC molecular weight. In interactions involving alkanes, the only Van der Waals forces present are London forces. As the alkane solute molecular surface area increases, London forces between the solute molecules increase in strength due to enhanced contact points making it more difficult to break the solute-solute interactions for solute-solvent interactions to occur. The branching of alkanes makes molecules compact and this reduces the effective contact surface area, thereby reducing the effect of London forces, making solute-solvent interactions easier Because triethylene glycol contains two primary alcohol groups, hydrogen bonding (very strong dipole-dipole Van der Waals forces) will dominate between solvent-solvent interactions. These attractive forces are much stronger than London forces and thus the solvent molecules will prefer to interact amongst themselves rather than with the alkane solutes, hence the high infinite dilution activity coefficients obtained. Reference [12] reported infinite dilution activity coefficients of volatile organic compounds in triethylene glycol. The infinite dilution activity coefficient for nonane was 182.6 at $50^{\circ} \mathrm{C}$ [12] compared to 63.3 found in this work at $30^{\circ} \mathrm{C}$. Similarly the experimentally obtained infinite dilution activity coefficients reported in the literature [12] of heptane (93.2555) and octane (129.3399) were much higher than the UNIFAC obtained values reported in this work. Sun et al. [12] found that in order for the Modified UNIFAC Dortmund results to correlate with experimental findings, the triethylene glycol solvent molecule would have to be treated as a special group with its own group interaction parameter values. It is therefore acknowledged that the results obtained in this work are only useful for the purposes of establishing general trends and thus only serve as a general indication of whether the solvent will be suitable as a scrubbing solvent. It is evident that Modified UNIFAC Lyngby dramatically under-predicts infinite dilution activity coefficients for alkane/ triethylene glycol interactions in comparison to Modified UNIFAC Dortmund.

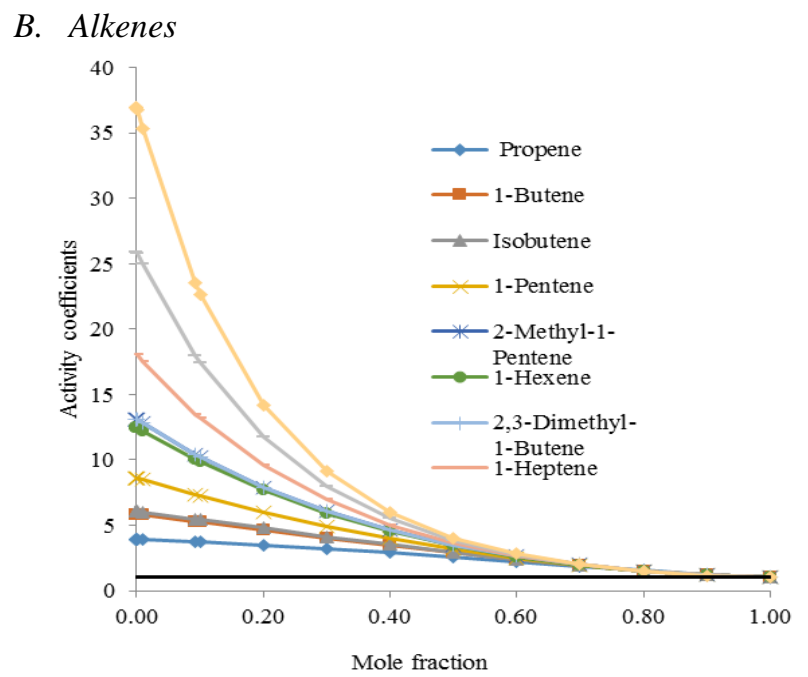

Fig. 3. Variation of Infinite dilution activity coefficients with mole fraction (Modified UNIFAC Dortmund).

Alkenes are more compact compared to alkanes of similar length or size and therefore require less energy to overcome the London forces of solute-solute interactions for solute-solvent bonding to occur. This could be the reason for 
the slightly increased solubility of the family in triethylene glycol when compared to their alkane counterparts. As for alkanes, the only Van der Waals forces that dominate are London forces. Thus there is effectively no dipole-induced dipole interaction between the triethylene glycol/ alkene solute interactions and hence the solvent molecules will prefer to interact with themselves rather than bonding to the alkenes. This is the reason for the high activity coefficients experienced with these interactions, Fig. 3 and Fig. 4.

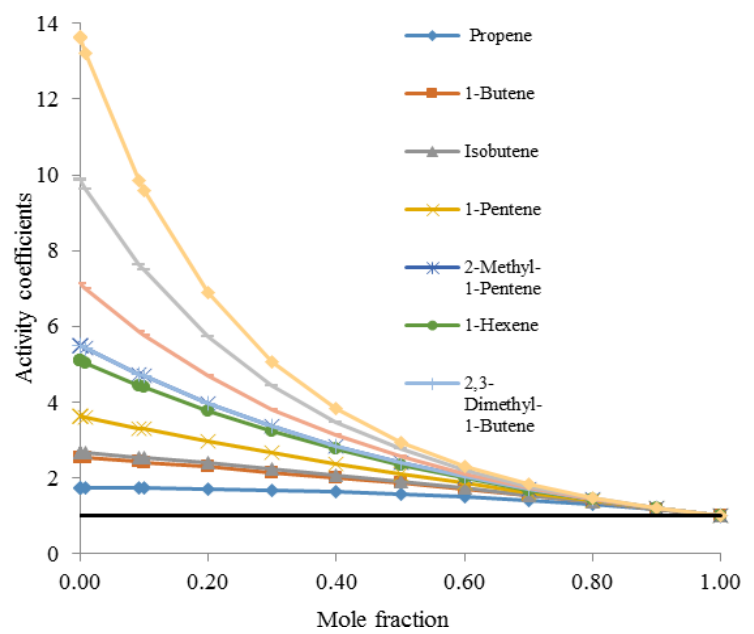

Fig. 4. Variation of Infinite dilution activity coefficients with mole fraction (Modified UNIFAC Lyngby).

C. Alkynes

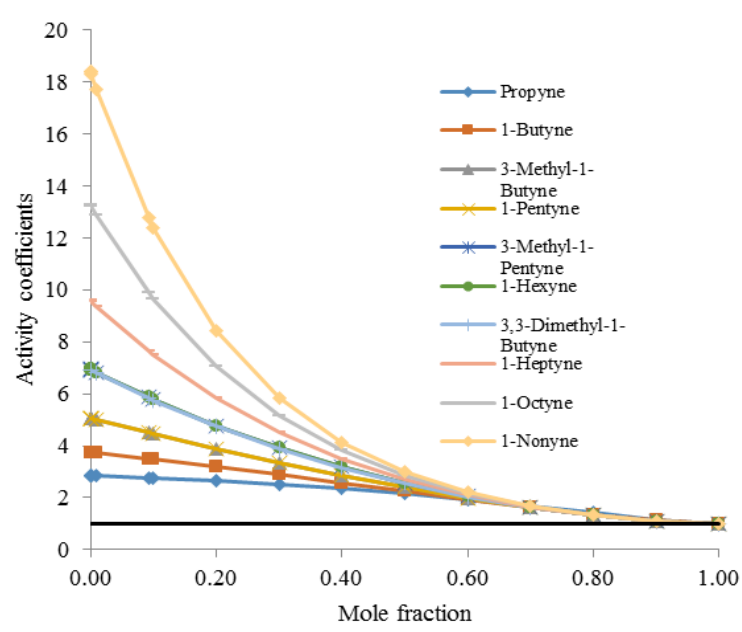

Fig. 5. Variation of Infinite dilution activity coefficients with mole fraction (Modified UNIFAC Dortmund).

Unlike alkanes and alkenes, alkynes have a triple unsaturated bond which is highly polarisable. It is therefore possible for the highly polar triethylene glycol solvent molecules to induce a dipole at the site of the triple bond, resulting in the alkyne solute-solute interactions breaking down to form solute-solvent bonds. The resultant attractive Van der Waals force is known as a Debye induction force. The above relationship is particularly true for the smaller alkynes. However as the triple bonds become increasingly shielded by the increase in size of the methylene tail, the behaviour of the alkyne approaches that of the alkanes where the London forces between the solute-solute interactions require much energy to break for solute-solvent bonding to occur, Fig. 5 and Fig. 6.

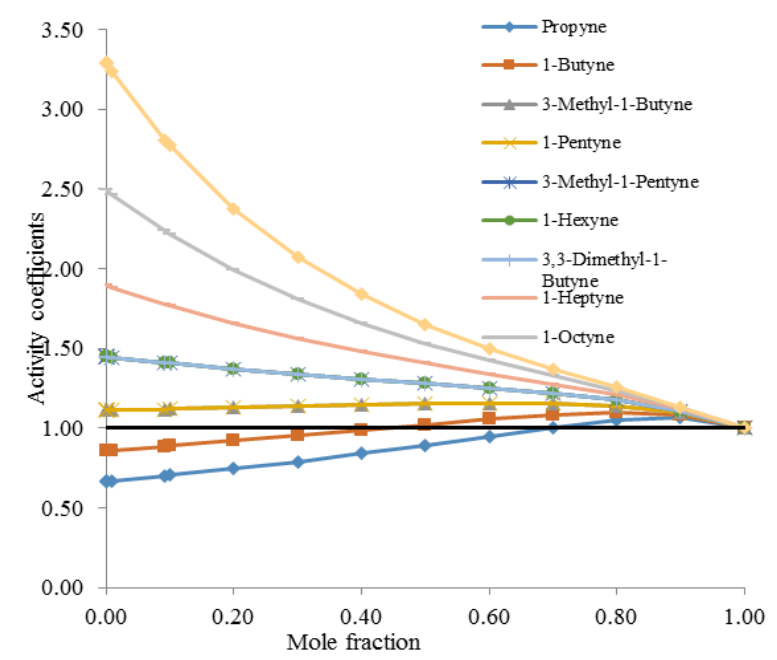

Fig. 6. Variation of Infinite dilution activity coefficients with mole fraction (Modified UNIFAC Lyngby).

\section{Aldehydes}

Aldehydes contain a carbonyl group with a powerful electron-attractive inducting effect. The presence of the carbonyl group makes the aldehydes polar in nature; therefore dipole-dipole (Keesom) interaction is possible between the aldehyde solutes and the polar solvent molecules. This makes aldehydes much more soluble in triethylene glycol than the alkanes, alkenes and alkynes. However aldehydes are only hydrogen bond acceptors because they lack a hydrogen atom bonded to their $\mathrm{C}=\mathrm{O}$ bond. Thus they are not as polar as triethylene glycol molecules and hence the solvent molecules would much prefer bonding with each other rather than with the aldehyde solutes. Solubility of aldehydes in triethylene glycol is reduced with an increase in the length of the methylene tail due to the increased shielding effect of the lengthening tail on the carbonyl group. Thus the solubility of aldehydes increases with a decrease in the VOC carbon number, Fig. 7. The Modified UNIFAC Lyngby could not reliably predict the phase equilibrium involving aldehydes and triethylene glycol, possibly because infinite dilution activity coefficients were not used in the fitting of the temperature dependent group interaction parameters $\left(\psi_{\mathrm{nm}}\right)$ [13].

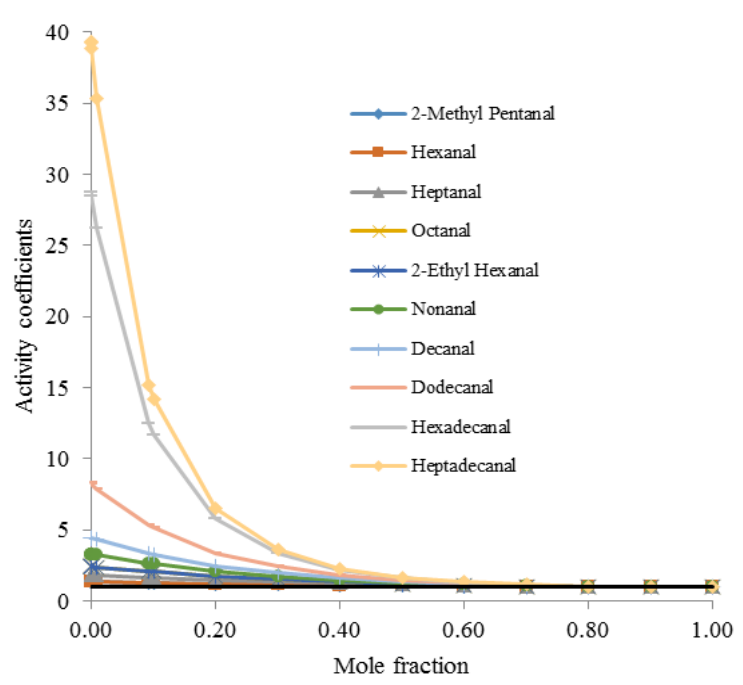

Fig. 7. Variation of Infinite dilution activity coefficients with mole fraction (Modified UNIFAC Dortmund). 


\section{E. Alcohols}

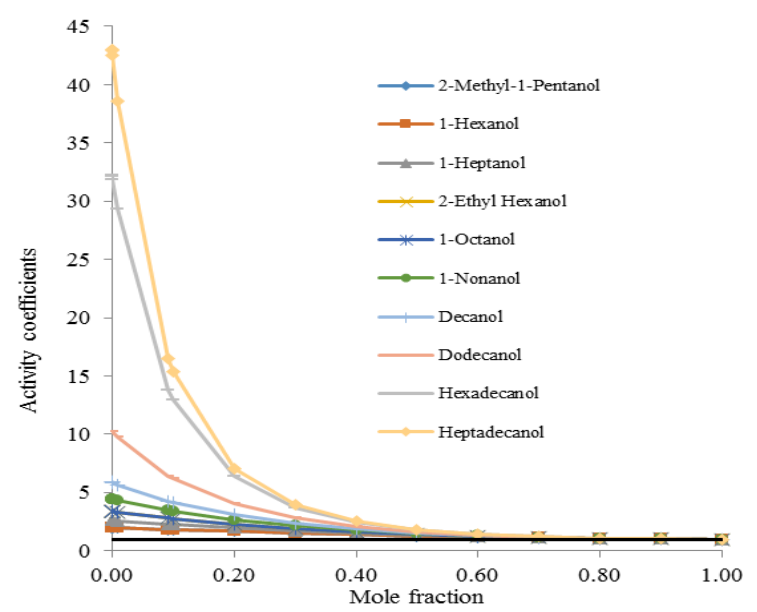

Fig. 8. Variation of Infinite dilution activity coefficients with mole fraction (Modified UNIFAC Dortmund).

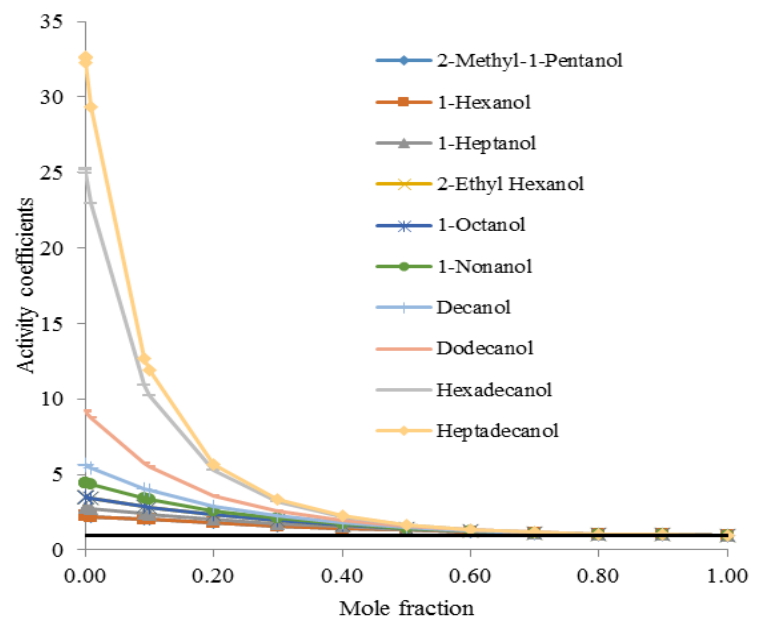

Fig. 9. Variation of Infinite dilution activity coefficients with mole fraction (Modified UNIFAC Lyngby).

With the exception of high molecular weight alcohols Fig. 8 and Fig. 9, most alcohols exhibited excellent solubility in triethylene glycol with infinite dilution activity coefficients below 5 . As for the solvent molecules, alcohols also contain a hydroxyl $(\mathrm{OH})$ group and hence also participate in hydrogen bonding. Thus solute-solute bonds and solvent-solvent bonds break readily to form solute-solvent bonds. With an increase in the length of the alcohol solute hydrocarbon methylene tail the effects of the hydrogen bonding site are shielded by the non-polar tail, and London forces between solute-solute interactions become stronger. This results in solute-solute interactions becoming increasingly difficult to break and hence solubility decreases with an increase in high molecular weight alcohols.

\section{F. Carboxylic Acids}

Carboxylic acids have a carbonyl group and an $\mathrm{OH}$ group. This makes carboxylic acids highly polar, since the hydroxyl group act as hydrogen bond donors and acceptors whilst the carboxyl group acts as hydrogen bond acceptors. Since triethylene glycol has two hydrogen bonding sites, and carboxylic acids also have two hydrogen bonding sites, solute-solvent bonding occurs readily. As a rule of thumb "Like dissolves like", hence the high solubility of carboxylic family of VOCs in triethylene glycol, Fig. 10 and Fig. 11.

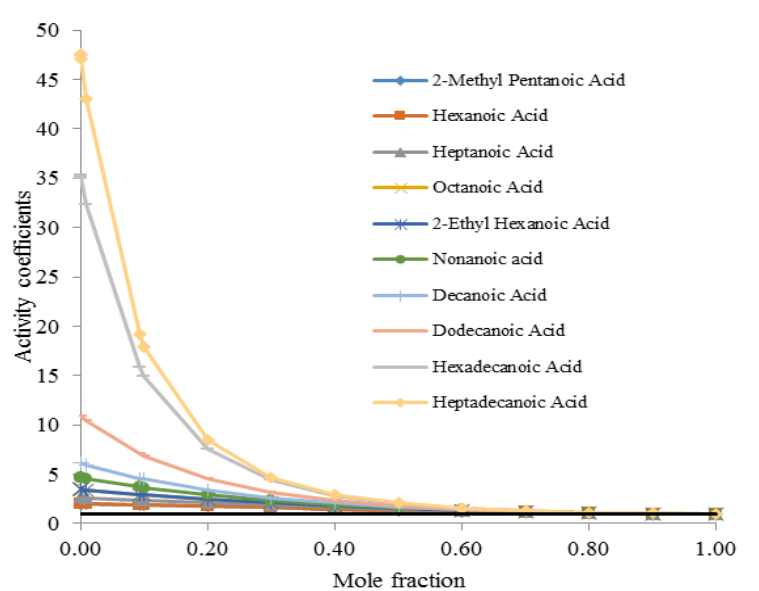

Fig. 10. Variation of Infinite dilution activity coefficients with mole fraction (Modified UNIFAC Dortmund).

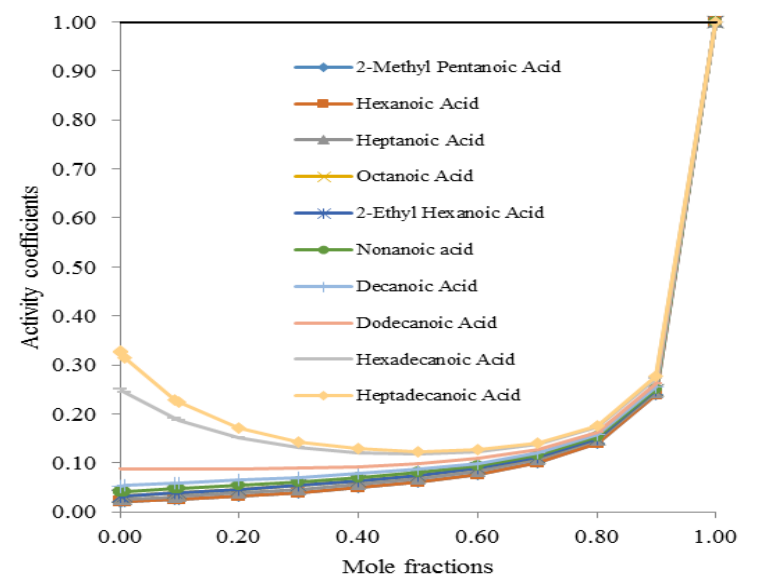

Fig. 11. Variation of Infinite dilution activity coefficients with mole fraction (Modified UNIFAC Lyngby).

\section{CONCLUSION}

This work attempted to predict the solubility of 60 selected VOCs in triethylene glycol using the Modified UNIFAC Dortmund and Lyngby models. The modified UNIFAC Lyngby gave unreliable results mainly due to the influence of the temperature dependent interaction parameters as well as the unavailability of the required parameters for most functional groups. The Modified UNIFAC Dortmund was more reliable in predicting the required phase equilibrium data; however the accuracy of both models is questionable without the development of a special group for the solvent with its own group interaction parameters. Based on the findings of this work triethylene glycol can be used for the physical absorption of volatile organic compounds from contaminated effluent air streams.

\section{ACKNOWLEDGMENT}

The authors acknowledge Miss Tintshwalo Precious Mathebula for performing the calculations and Mr. Jacques J Scheepers for critically evaluating the manuscript. The Universities of Johannesburg and South Africa are also acknowledged for financial support.

\section{REFERENCES}

[1] F. I. Khan and A. K. Ghoshal, "Removal of volatile organic compounds from polluted air," Journal of Loss Prevention in the Process Industries, vol. 13, pp. 527-545, 2000. 
[2] E. C. Voutsas and D. P. Tassios, "Prediction of infinite-dilution activity coefficients in binary mixtures with UNIFAC. A critical evaluation," Ind. Chem. Chem. Res., vol. 35, pp. 1438-1445, 1986.

[3] K. Bay, H. Wanko, and J. Ulrich, "Absorption of volatile organic compounds in biodiesel: Determination of infinite dilution activity coefficients by headspace gas chromatography," Chem. Eng. Res. Des., vol. 84, issue 1, pp. 22-27, Jan. 2006.

[4] K. Bay, H. Wanko, and J. Ulrich, "Biodiesel - Hoch siedendes absorbens für die Gasrienigung," Chemie Ingenieur Technik, vol. 76, no. 3, pp. 328-333, Mar. 2004

[5] U. Weidlich and J. Gmehling, "A modified UNIFAC model. 1. Prediction of VLE, hE, and $\gamma^{\infty}$," Ind. Eng. Chem. Res., vol. 26, no. 7, pp. 1372-1381, Jul. 1987.

[6] J. J. Scheepers, E. Muzenda, and M Belaid, "Influence of temperature and molecular structure on organics-biodiesel interactions using group contribution methods," in Proc. World Congress on Engineering 2012 IAENG, London, UK, 4-6 July 2012, vol. III.

[7] J. J. Scheepers, E. Muzenda, and M. Belaid, "Influence of structure on fatty acid ester-alkane interactions," in Proc. International Conference on Chemical Engineering and its Applications, Bangkok, Sept. 2012, pp. 93-102.

[8] J. J. Scheepers and E. Muzenda, "Alkenes - ester polymeric solvents thermodynamic interactions - Part 1," in Proc. International Conference of the Planetary Scientific Research Centre, 2012, pp. 229-232.

[9] J. J. Scheepers, E. Muzenda, and M. Belaid, "Alkenes - ester polymeric solvents thermodynamic interactions - Part 2," in Proc. International Conference of the Planetary Scientific Research Centre, 2012, pp. 54-57.

[10] J. J. Scheepers, E. Muzenda, and M. Belaid, "Intermolecular dynamics between aromatic compounds and ester polymeric solvents," in Proc. International Conference of PSRC, 2013, pp. 37-41.

[11] E. Muzenda, M. Belaid, and F. Ntuli "Measurement of infinite dilution activity coefficients of selected environmentally important volatile organic compounds in polydimethylsiloxane using gas - liquid chromatography," The Korean Journal of Chemical Engineering, vol. 27, no. 5, pp. 1509-1512, 2010.

[12] P. P. Sun, G. H. Gao, and H. Gao, "Infinite dilution activity coefficients of hydrocarbons in triethylene glycol and tetraethylene glycol," Ind. Chem. Chem. Res., vol. 48, pp. 1109-1112, 2003.

[13] J. Gmehling, J. Li, and M. Schiller, "A modified UNIFAC model. 2. present parameter matrix and results for different thermodynamic properties," Ind. Eng. Chem. Res., vol. 32, pp. 178-193, 1993.

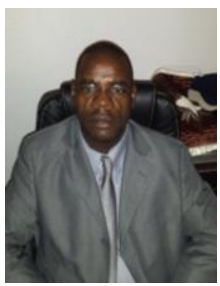

Edison Muzenda is a full professor of chemica engineering, he is a research and postgraduate coordinator as well as the head of the Environmental and Process Systems Engineering Research Group in the Department of Chemical Engineering at the University of Johannesburg. Professor Muzenda holds a BSc Hons degree (ZIM, 1994) and a PhD degree in chemical engineering (Birmingham, 2000). He has more than 15 years' experience in academia which he gained at different Institutions: National University of Science and Technology, University of Birmingham, Bulawayo Polytechnic, University of Witwatersrand, University of South Africa and the University of Johannesburg. Through his academic preparation and career, Edison has held several management and leadership positions such as member of the student representative council, research group leader, university committees' member, staff qualification coordinator as well as research and postgraduate coordinator. Edison's teaching interests and experience are in unit operations, multi-stage separation processes, environmental engineering, chemical engineering thermodynamics, entrepreneurship skills, professional engineering skills, research methodology as well as process economics, management and optimization. He is a recipient of several awards and scholarships for academic excellence. His research interests are in green energy engineering, integrated waste management, volatile organic compounds abatement and as well as phase equilibrium measurement and computation. He has published more than 180 international peer reviewed and refereed scientific articles in journals, conferences and books. Edison has supervised 28 postgraduate students, 4 postdoctoral fellows as well as more than 140 Honours and BTech research students. He serves as reviewer for a number of reputable international conferences and journals. Edison is a member of the Faculty of Engineering and Built Environment Research and Process, Energy and Environmental Technology Committees. He has also chaired several sessions at International Conferences. Edison is an associate member of the Institution of Chemical Engineers (AMIChemE), member of the International Association of Engineers (IAENG); associate member of Water Institute of Southern Africa (WISA), associate editor for the South African Journal of Chemical Engineering as well as a member of the Scientific Technical Committees and Editorial Boards of several scientific organizations.

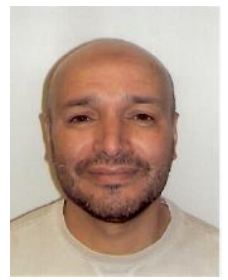

Mohamed Belaid obtained MSc degree in chemical engineering, UKZN South Africa (2001), BSc degree in industrial chemical engineering, engineering of organic processes (1994), University of Blida, Algeria. Currently is doing $\mathrm{PhD}$ studies at Wits University (South Africa), project title: "Behavior of selected SA coals in circulating Fluidised bed," Mohamed is employed at the University of Johannesburg as the head of Department of Chemical Engineering and a senior lecturer, he worked as a lecturer at the University of Kwa-zulu Natal for over 8 years, a quality control engineer for Energy Engineering PTY (South Africa) for two years and Elangeni oil and soap (South Africa) for a period of two years, process engineer (SAIDAL, antibiotic company, Algeria) for one year. Mohamed is a member of SAICHE (2003, South Africa institute of Chemical Engineers). He has authored and contributed to various publications both journals and conferences proceedings in environmental engineering, separation processes, mineral processing, fluidized beds, activated carbon and engineering education.

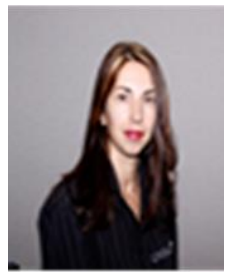

Corina Maria Mateescu holds a bachelor degree of engineering (chemical) from University of "Babes-Bolyai". She is currently working for a master degree in chemical engineering at the University of Johannesburg under the supervision of Prof E. Muzenda. She is a lecturer in Chemical Engineering Department at the University of South Africa (UNISA) since Jan 2010. Previously she worked as a lecturer at University of Johannesburg from July 2006 -31st December 2009. She also taught chemistry and physics at a secondary school. Her research interests are in phase equilibrium measurement and computation using group contributions methods, energy and environment. Mrs Mateescu is a team leader of a Community Engagement Project-Gir Power a project to enable, educate, and motivate girls to explore, enjoy and become more proficient in mathematics, science and technology. She is also a member of Southern African Society for Cooperative Education. 